FORSCHUNGSZENTRUM ROSSENDORF $374 R$

\author{
FZR-209 \\ Januar 1998 \\ Preprint
}

K. Gallmeister, B. Kämpfer und O. P. Pavlenko

Thermal dilepton signal versus dileptons from open charm and bottom decays in heavy-ion collisions

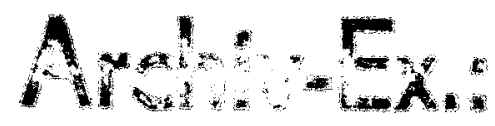


Herausgeber:

FORSCHUNGSZENTRUM ROSSENDORF

Postfach 510119

D-01314 Dresden

Telefon (03 51) 2600

Telefax (03 51) 2690461

Als Manuskript gedruckt

Alle Rechte beim Herausgeber 


\title{
Thermal dilepton signal versus dileptons from open charm and bottom decays in heavy-ion collisions
}

\author{
K. Gallmeister ${ }^{a}$, B. KÄMPfeR ${ }^{a}$, O.P. PAVlenko ${ }^{a, b}$ \\ ${ }^{a}$ Research Center Rossendorf, PF 510119, 01314 Dresden, Germany \\ ${ }^{b}$ Institute for Theoretical Physics, 252143 Kiev - 143, Ukraine
}

\begin{abstract}
We analyze the opportunity to observe thermal dileptons emitted off deconfined matter resulting in heavy-ion collisions at RHIC and LHC energies. Special kinematical conditions provided by the detector systems PHENIX and ALICE, and the so-called $M_{\perp}$ scaling behavior of thermal dilepton spectra are taken into account. Our considerations include energy loss effects of the fast heavy quarks in deconfined matter, which for themselves can help to identify the creation of a hot and dense parton medium. Due to a threshold like effect for decay leptons we find a window at large transverse pair momentum and fixed transverse mass where the thermal signal can exceed the background of dileptons from correlated semileptonic decays of charm and bottom mesons.
\end{abstract}

\section{INTRODUCTION}

One of the ultimate goals in investigating central heavy-ion collisions at very high energies is to analyze the properties of highly excited and deconfined matter. Direct probes, like electromagnetic signals [1], are considered as useful carriers of nearly undisturbed information from the transient hot reaction stages. Pairs of electrons and muons are experimentally accessible, and will be measured also in forthcoming heavy-ion experiments [2,3]. These types of probes have proven useful at Super Proton Synchrotron (SPS) energies at CERN in the resonance region below the $\omega, \rho[4]$, and in the $J / \psi$ region [5]; also in the so-called continuum region in between a yet unexplained excess seems to be found [6]. However, recent estimates [7] of dileptons stemming from semileptonic decays of open charm and bottom mesons produced in the same central collisions of heavy ions show that these represent the dominating dilepton source at energies envisaged at the Relativistic Heavy Ion Collider (RHIC) in Brookhaven and at the Large Hadron Collider (LHC) in CERN. In particular, the dilepton signal from ther- 
malized, strongly interacting matter in the intermediate reaction stages is estimated to be up to two orders of magnitude below the background of correlated decays of open charm or bottom meson pairs in a wide invariant mass region. Even strong energy loss effects of the heavy quarks in the hot and dense medium [8-10] do not basically change this situation: The decay dileptons might be suppressed down to the Drell-Yan background, but it is questionable whether such strong energy loss effects can happen in reality [11]. In this situation at least two issues can be seen. First, it is necessary to extend the traditional strategy for searching a thermal dilepton signal from deconfined matter. The usual approach relies on the analysis of the invariant mass $(M)$ spectra. One can expect, however, that the double differential dilepton spectrum as a function of the transverse pair momentum $Q_{\perp}$ and transverse pair mass $M_{\perp}=\sqrt{M^{2}+Q_{\perp}^{2}}$ contains much more information which can be used to impose special kinematical gates for finding a window to observe the thermal dileptons in the continuum region in spite of the large decay background. Second, one can try to employ the initial hard production of open charm and bottom followed by semileptonic decays to probe deconfined matter by energy loss effects. A similar idea for hard jet production is widely under discussion now [12].

In the present paper we focus on both of the above issues. We compare the so-called $M_{\perp}$ scaling property of the dielectrons stemming from deconfined matter with pairs resulting from correlated open charm and bottom decays. As well-known, under certain conditions the dilepton yield from a thermalized quark-gluon plasma depends only on the transverse mass, i.e., there is no dependence on the variable $Q_{\perp}$ at fixed $M_{\Perp}$ in a given rapidity interval $Y$. At the same time the corresponding $Q_{\perp}$ dependence of dileptons from heavy quark decays has qualitatively a different behavior: It is peaked at small values of $Q_{\perp}$ and drops rapidly at increasing values of the transverse momentum. To get realistic estimates of the $M_{\perp}$ scaling we perform Monte Carlo simulations for both the correlated heavy quark decays and the thermal production including the acceptance of the detectors PHENIX at RHIC and ALICE at LHC. To strengthen the $M_{\perp}$ scaling violation for dileptons from decays we select only such leptons with individual transverse momentum $p_{\perp}>p_{\perp}^{\min }$ with appropriate value of $p_{\perp}^{\min }$. We find that, while the thermal dilepton yield from the plasma is less affected by the kinematical restriction, the contributions from decays at large transverse pair momentum is reduced drastically. Actually this leads to a threshold like behavior of the transverse momentum spectrum at fixed value of $M_{\perp}$ for dileptons from heavy quark decays in contrast to the approximate plateau of the thermal yield which extends up to the kinematical limit at 
$Q_{\perp}=M_{\perp}$. As a result, an opportunity is found which allows to separate the thermal dilepton signal from the background of heavy quark decays on the basis of the $M_{\perp}$ scaling property. We also study to what extent the single electron and dilepton spectra are modified by the energy loss effects of heavy quarks when traversing deconfined matter. In doing so we concentrate on calculable processes in the first few $\mathrm{fm} / \mathrm{c}$ of the collision course. There are, of course, other processes in the later reaction stages that might considerably affect the momenta of the charm and bottom mesons [13], and also thermal radiation of semihard probes can stem from later stages. For instance, in ref. [14] the hypothesis has been formulated, resting on an analysis of present $\mathrm{Pb}+\mathrm{Pb}$ data at SPS energy, that all hadron spectra can be parametrized by a common flow velocity and freeze-out temperature. This, if correct for higher energies too, would imply that charm and bottom spectra are much stronger modified than assumed in the following, and as a consequence the chances to identify a thermal dilepton source would be better.

Our paper is organized as follows. In section 2 we present our calculation procedure of charm and bottom spectra and the resulting single electron and dielectron spectra. Section 3 is devoted to the thermal dileptons at midrapidity and the $M_{\perp}$ scaling properties. Our conclusions can be found in section 4 .

\section{DILEPTONS FROM OPEN CHARM AND BOTTOM DECAYS}

\section{A. Initial charm and bottom production}

In our approach we utilize the leading order QCD processes $g g \rightarrow Q \bar{Q}$ and $q \bar{q} \rightarrow Q \bar{Q}$ for heavy quark - antiquark $(Q \bar{Q})$ production and simulate higher order corrections by an appropriate $\mathcal{K}_{Q}$ factor. We have checked that such a procedure reproduces within the needed accuracy the more involved next-to-leading order calculations [7]. The number of $Q \bar{Q}$ pairs, produced initially with transverse momenta $p_{\perp 1}=-p_{\perp 2}=p_{\perp}$ at rapidities $y_{1,2}$ in central $A A$ collisions can be calculated by

$$
\begin{aligned}
d N_{Q \bar{Q}}= & T_{A A}(0) \mathcal{K}_{Q} H\left(y_{1}, y_{2}, p_{\perp}\right) d p_{\perp}^{2} d y_{1} d y_{2}, \\
H\left(y_{1}, y_{2}, p_{\perp}\right)= & x_{1} x_{2}\left\{f_{g}\left(x_{1}, \hat{Q}^{2}\right) f_{g}\left(x_{2}, \hat{Q}^{2}\right) \frac{d \hat{\sigma}_{g}^{Q}}{d \hat{t}}\right. \\
& \left.+\sum_{q \tilde{q}}\left[f_{q}\left(x_{1}, \hat{Q}^{2}\right) f_{\tilde{q}}\left(x_{2}, \hat{Q}^{2}\right)+f_{q}\left(x_{2}, \hat{Q}^{2}\right) f_{\tilde{q}}\left(x_{1}, \hat{Q}^{2}\right)\right] \frac{d \hat{\sigma}_{q}^{Q}}{d \hat{t}}\right\}
\end{aligned}
$$

where $\hat{\sigma}_{q, g}^{Q} / d \hat{t}$ are elementary cross sections (see for details $\left.[7,15]\right), f_{i}\left(x, \hat{Q}^{2}\right)$ with $\dot{i}=$ $g, q, \bar{q}$ denote the parton structure functions, $x_{1,2}=m_{\perp}\left(\exp \left\{ \pm y_{1}\right\}+\exp \left\{ \pm y_{2}\right\}\right) / \sqrt{s}$ 
and $m_{\perp}=\sqrt{p_{\perp}^{2}+m_{Q}^{2}}$. As heavy quark masses we take $m_{c}=1.5 \mathrm{GeV}$ and $m_{b}=$ 4.5 GeV. We employ the HERA supported structure function set MRS D'- [16] from the PDFLIB at CERN. Nuclear shadowing effects are not included since they will be considered separately. In addition, for our present goals we expect no significant modification by shadowing in the large transverse momentum region according to results of ref. [17]. The overlap function for central collisions is $T_{A A}(0)=A^{2} /\left(\pi R_{A}^{2}\right)$ with $R_{A}=1.1 A^{1 / 3} \mathrm{fm}^{\text {a }}$ and $A=200$. From a comparison with results of ref. [7] we find the scale $\hat{Q}^{2}=4 M_{Q}^{2}$ and $\mathcal{K}_{Q}=2$ as most appropriate.

\section{B. Energy loss effects}

Energy loss effects (cf. [11,18-21] and further references therein) have been found important [8-10] since the momenta of back-to-back moving heavy quarks are degraded and the resulting dileptons in correlated semileptonic decays get less invariant mass. This process causes a considerable reduction of the number of high invariant mass dileptons from correlated $D \bar{D}$ and $B \bar{B}$ decays.

To model the energy loss effects of heavy quarks in expanding matter we assume that a heavy quark has the same rapidity as the longitudinal flow at the space-time point of the initial quark production. Therefore, with respect to the fluid's local rest frame the heavy quark has essentially only a transverse momentum $p_{\perp}$ which may depend on the proper local time $\tau$ in accordance with the energy loss $d E / d x$ in transverse direction. This picture implies a very fast thermalization of gluons and light quarks.

Unfortunately a sufficiently general treatment of the energy loss rate $d E / d x$ for heavy quarks passing through expanding QCD matter has not been developed so far. Recently the total energy loss of a high-energy parton propagating transversely through expanding deconfined matter has been derived as $d E /\left.d x\right|_{\text {expanding }}=\xi d E /\left.d x\right|_{T_{f}}[11]$, supposed the medium cools according to a power law and the initial time is short. The numerical factor $\xi$ is $\mathcal{O}(2)$ for a parton created inside the medium. Within such a scenario the stopping power depends only on the final temperature at which the deconfined medium is left, as indicated by the subscript $T_{f}$. We denote this stopping scenario as model I and take $d E /\left.d x\right|_{T_{f}}$ from [11,19-21]. This model is appropriate for LHC conditions, where the initial temperature is very high and where the life time of deconfined matter is large enough that most energetic heavy quarks can leave this stage before $\mathcal{T}_{c}$ is reached. For RHIC conditions we adopt the results of ref. [19] for $d E / d x$ in a static medium but apply them in the simplest generalization to a medium with time 
dependent density. This gives evolution equations for the transverse momentum $p_{\perp}(\tau)$ of quarks propagating the distance $r_{\perp}(\tau)$ in the transverse direction. The scenario is denoted as model II, which is described in more detail in refs. [9,14]. Strictly speaking, due to the finite size of the medium, the large mass of the heavy quarks and other approximations inherent in such approaches, the models I and II provide at best semiquantitative estimates. Nevertheless one can hope to cover various limiting cases to get some insight in the importance of the energy loss effects.

To get the spectra of charm and bottom quarks after energy loss we use a Monte Carlo simulation with a uniform distribution of the random initial position and random orientation of $Q \bar{Q}$ pairs in the transverse plane. In model I the total energy loss is determined by the transverse distance $d$ of a created heavy quark to the boundary of the system, the quark initial energy and the temperature when leaving the system. Most quarks experience an energy loss $\propto d$ and only a few ones $\propto d^{2}$. (The transverse expansion at early times can be neglected, and we take as averaged transverse radius of parton matter $R=7 \mathrm{fm}$.) In model II we integrate the evolution equations for the heavy quark momentum $p_{\perp}(\tau)$ and position $r_{\perp}(\tau)$ together with the time evolution of the temperature $T(\tau)$ and fugacities $\lambda_{q, g}(\tau)$ (see below) as described in refs. [9,15]: The quantity $r_{\perp}(\tau)$ is used to check whether the considered heavy quark propagates still within deconfined matter; if it leaves the deconfined medium it does not longer experience an energy loss. (The energy loss in a mixed and hadron phase might be incorporated in line with the approach of [13].) We consider a heavy quark to be thermalized if its transverse mass $m_{\perp}$ during energy loss becomes less than the averaged transverse mass of thermalized light partons at given temperature. These heavy quarks are redistributed according to the transverse mass distribution $d N_{Q} / d m_{\perp} \propto m_{\perp}^{2} \exp \left\{-m_{\perp} / T_{\text {eff }}\right\}$ with an effective temperature $T_{\text {eff }}=150 \mathrm{MeV}[10]$.

\section{Single electron spectra}

We employ a delta function like fragmentation scheme for the heavy quark conversion into $D$ and $B$ mesons. It results in the same transverse momentum of the heavy meson as the heavy parent quark has had previously. Inclusive single transverse momentum spectra of electrons/positrons from semileptonic open charm and bottom decays, i.e., $D(B) \rightarrow e^{+} X$, and dilepton spectra from correlated decays as well, i.e., $D(B) \bar{D}(\bar{B}) \rightarrow e^{+} X e^{-} \bar{X}$, are obtained from a Monte Carlo code which utilizes the inclusive primary electron energy distribution as delivered by JETSET 7.4. The heavy 
mesons are randomly decayed in their rest system and the resulting electrons then boosted appropriately. The average branching ratio of $D(B) \rightarrow e^{+} X$ is taken as 12 (10)\% [22]. The neglect of secondary electrons is justified since we consider here single electron spectra with $p_{\perp}>1 \mathrm{GeV}$ and dilepton spectra with $M>2 \mathrm{GeV}$. The average energies of the secondary electrons, in particular from $B$ decays, are too small to affect the spectra in these kinematical regions noticeable.

The following acceptance cuts are utilized in our Monte Carlo calculations. PHENIX [2] can register electrons in the pseudorapidity window $|\eta| \leq 0.35$ with azimuthal coverage $0-90$ and 135 - 225 degrees, while ALICE [3] is azimuthally symmetric and covers $|\eta| \leq 0.9$. As minimum transverse electron momentum we use $p_{\perp}=1 \mathrm{GeV}$. A maximum transverse momentum cut is not imposed, even at ALICE there is probably one. We consider here only the electron and $e^{+} e^{-}$channels and the midrapidity region since the energy loss effects are strongest there [10].

The results of our calculations of the transverse momentum spectra of inclusive single electrons at RHIC conditions are depicted in figs. 1a and b. As already pointed out in ref. [23], a measurement of single electrons with $p_{\perp}>1.5 \mathrm{GeV}$ at RHIC can give a clean and background-free charm signal in heavy-ion collisions. Energy loss effects of the heavy quarks modify this signal. As seen in figs. 1a and b such modifications, according to the energy loss described above, are measurable. For both the charm and the bottom decay the transverse single electron spectra become softer. This leads to a noticeable change of the slope of the spectra. One can fit the distributions by $d N_{e^{-}} / d p_{\perp} \propto \exp \left\{-p_{\perp} / T_{e}\right\}$ in the interval $2.5 \mathrm{GeV}<p_{\perp}<4.5 \mathrm{GeV}$ and finds $T_{e}=$ 646 (512) $\mathrm{MeV}$ in case of no stopping (stopping according to model II) for charm, and $T_{e}=851(640) \mathrm{MeV}$ for bottom. The superposition of charm and bottom decay spectra can be parametrized by $T_{e}=711$ (574) $\mathrm{MeV}$. Therefore, in comparing the $p_{\perp}$ spectra of single electrons in pp and AA collisions at the same beam energies one can reveal the stopping effect. (A direct measurement of the $D(B) p_{\perp}$ distributions would be more useful, but it is unclear whether the $D(B)$ mesons can be identified via their hadronic decay modes in such high-multiplicity environments.) In extreme case, when the stopping is so large that thermalization with the surrounding matter happens, then a pronounced "thermal bump" at $p_{\perp}<2.5 \mathrm{GeV}$ appears. All of these considerable modifications of the transverse electron spectra in AA collisions in comparison with pp collisions can be helpful for identifying the creation of a hot and dense parton system. It should be noted that the energy loss effects affect the single-electron $p_{\perp}$ spectra much stronger than another fragmentation scheme (e.g. the below described Peterson 
scheme) would do.

\section{Decay dielectrons}

The invariant mass dilepton spectra resulting from both correlated charm and bottom decays are displayed in figs. $2 \mathrm{a}$ and $\mathrm{b}$ for RHIC and LHC energies. Also shown is the distribution of thermal dileptons according to eq. (9) below. As pointed out earlier [7] the $M$ spectra are by far dominated by the dileptons from correlated open charm and bottom decays. A strong energy loss according to the above models reduces the decay contributions, so that (i) at RHIC charm and bottom decay dileptons are in the same order of magnitude as the Drell-Yan background [9,10], and (ii) at LHC still bottom will dominate the Drell-Yan background, and charm is slightly below bottom [9]. While without any detector acceptances usually the predictions of the thermal yield (dealt with in the next section) is in the same order of magnitude as the DrellYan background $[7,9,15,24]$, it turns out that both the acceptances of PHENIX and ALICE suppress the thermal signal stronger than the Drell-Yan background. At the first sight such a situation looks quite unfavorable for a measurement of the thermal signal even at such high energies as achieved at RHIC and LHC. Nevertheless we show below that one can select special kinematical conditions superimposed to the detector cuts to analyze the more informative double differential dilepton spectrum with the aim to find a window for the thermal signal.

\section{THERMAL DILEPTON PRODUCTION AND $M_{\perp}$ SCALING}

\section{A. Spectra of thermal dileptons}

In order to estimate the thermal dilepton yield from deconfined matter we restrict ourselves to the lowest order processes $q \bar{q} \rightarrow \gamma^{*} \rightarrow e^{+} e^{-}$, i.e., the electromagnetic annihilation of quarks and antiquarks. Contributions from QCD processes like $q \bar{q} \rightarrow$ $g e^{+} e^{-}$and $q g \rightarrow q e^{+} e^{-}$have been considered [24] and appear to be not very significant (typically they increase the yield by a factor less than 2.5). The rate of dilepton production per space-time volume can be obtained within a kinetic theory approach as

$$
\begin{aligned}
\frac{d N_{e^{+} e^{-}}}{d^{4} x}= & \int \frac{d^{3} p_{1}}{2 E_{1}(2 \pi)^{3}} \frac{d^{3} p_{2}}{2 E_{2}(2 \pi)^{3}} \frac{d^{3} p_{+}}{2 E_{+}(2 \pi)^{3}} \frac{d^{3} p_{-}}{2 E_{-}(2 \pi)^{3}} f_{1}\left(x, p_{1}\right) f_{2}\left(x, p_{2}\right) \\
& \times\left|\mathcal{M}\left(12 \rightarrow e^{+} e^{-}\right)\right|^{2}(2 \pi)^{4} \delta^{(4)}\left(p_{1}+p_{2}-p_{+}-p_{-}\right),
\end{aligned}
$$


where $p_{a}=\left(E_{a}, \vec{p}_{a}\right)$ are the four momenta of incoming quarks $(a=1,2)$ and outgoing leptons $\left(a=e^{+}, e^{-}\right)$. The quantity $\left|\mathcal{M}\left(12 \rightarrow e^{+} e^{-}\right)\right|^{2}$ denotes the square of the amplitude of the process, summed over initial and final state particles. The quantities $f_{a}\left(x, p_{a}\right)$ stand for the distribution functions of quarks and antiquarks. Integrating eq. (3) over the momenta of the leptons one gets a widely used expression [25] for the dilepton rate per four-momentum of the pair, $Q=p_{+}+p_{-}$,

$$
\frac{d N_{e^{+} e^{-}}}{d^{4} x d^{4} Q}=\int \frac{d^{3} p_{1}}{(2 \pi)^{3}} \frac{d^{3} p_{2}}{(2 \pi)^{3}} f_{1}\left(x, p_{1}\right) f_{2}\left(x, p_{2}\right) v \sigma\left(M^{2}\right) \delta^{(4)}\left(p_{1}+p_{2}-Q\right)
$$

The pair's four momentum can be expressed as $Q=\left(M_{\perp} \operatorname{ch} Y, \vec{Q}_{\perp}, M_{\perp} \operatorname{sh} Y\right)$ with $M_{\perp}=$ $\sqrt{M^{2}+Q_{\perp}^{2}}$ as transverse mass, $M$ as invariant mass, and $Y$ as rapidity of the pair. The relative quark velocity reads $v=M /\left(2 E_{1} E_{2}\right)$, while the total cross section is $\sigma\left(M^{2}\right)=\frac{4 \pi \alpha^{2}}{3 M^{2}} 12 F_{q}$ with quark charge factor $F_{q}=\sum e_{q}^{2}=\frac{5}{9}$ for $u, d$ quarks, and $\alpha$ is the fine structure constant.

For the calculation of dilepton spectra in the region of large invariant mass one can utilize the Boltzmann approximation for the incoming partons

$$
f_{a}(x, p)=\lambda_{a}(x) \exp \left\{\frac{p_{a} \cdot u(x)}{T(x)}\right\}
$$

where $u$ is the four-velocity of the medium. The above parton distributions are assumed to be in thermal equilibrium in momentum space, but not necessarily in chemical equilibrium. Only for chemical equilibrium the fugacities $\lambda_{a}$ become unity.

\section{B. $M_{\perp}$ scaling}

The space-time evolution of the produced deconfined matter is governed in our approach by the longitudinal scaling-invariant expansion accompanied by quark and gluon chemical equilibration processes $[24,26]$. Within such a model the space-time volume reads $d^{4} x=\pi R_{A}^{2} \tau d \tau d \tilde{\eta}$ with $\tau$ as proper time and flow rapidity $\tilde{\eta}$. Integrating the rate eq. (4) over the parton momenta and space-time evolution results in the dilepton spectrum

$$
\frac{d N_{e^{+} e^{-}}}{d M_{\perp}^{2} d Q_{\perp}^{2} d Y}=\frac{\alpha^{2} R_{A}^{2}}{4 \pi^{2}} F_{q} \int d \tau \tau K_{0}\left(\frac{M_{\perp}}{T(\tau)}\right) \lambda_{q}^{2}(\tau)
$$

The physical information encoded in the spectrum eq. (6) can be inferred from the following approximation. For large values of $M_{\perp}$ the main contribution to the time integral stems from early, hot stages at $\tau \sim \tau_{i}$. Under the assumption that the equation of state behaves like $p \propto e$ (here $p$ and $e$ stand for the pressure and energy density, 
respectively) one can derive from energy-momentum conservation the relation $T=$ $T_{i}\left(\tau_{i} / \tau\right)^{1 / 3} \hat{F}(\tau), \hat{F}=\left[\left(d_{g} \lambda_{i}^{g}+d_{q} \lambda_{i}^{q}\right) /\left(d_{g} \lambda^{g}(\tau)+d_{q} \lambda^{q}(\tau)\right)\right]^{1 / 4}$ with $d_{q, g}$ as degeneracy factors of quarks and gluons. Since near $\tau_{i}$ one can approximate $\hat{F} \approx 1$ and $\lambda^{q} \approx \lambda_{i}^{q}$, one arrives at

$$
\frac{d N_{e^{+} e^{-}}}{d Y d M_{\perp}^{2} d Q_{\perp}^{2}} \approx \frac{3 \alpha^{2} R_{A}^{2} F_{q}}{4 \pi^{2}}\left(\frac{\tau_{i} \lambda_{i}^{q} T_{i}^{3}}{M_{\perp}^{3}}\right)^{2} H\left(\frac{M_{\perp}}{T_{i}}\right)
$$

with $H(x)=x^{3}\left(8+x^{2}\right) K_{3}(x)$ and $K_{n}$ as modified Bessel function of $n$th order. For the possible initial conditions at LHC (see below) eq. (7) represents a fairly good approximation, also for small values of $\lambda_{i}^{q, g}$. Eq. (7) has the structure $\hat{f}_{1}\left(\tau_{i} \lambda_{i}^{q}\right) \hat{f}_{2}\left(M_{\perp} / T_{i}\right)$, therefore one can infer from it the value of $T_{i}$ by measuring the transverse rate at two distinct values of $M_{\perp}$. Afterwards, the combination $\tau_{i} \lambda_{i}^{q}$ can be extracted. If one could constrain by other means the initial time of the thermalized era, $\tau_{i}$, then even eq. (7) allows to estimate the initial fugacity $\lambda_{i}^{q}$. Due to particle production processes the comoving entropy density does not longer serve as a link between initial and final states in the evolution dynamics.

Our choice of initial conditions for produced deconfined matter is based on the estimates of refs. $[15,27]$ for the minijet plasma with a suitable parametrization of the soft component [9] which are similar to the self-screened parton cascade model [28]. We take as main set of parameters for the initial temperature $T_{i}=550(1000) \mathrm{MeV}$, for gluon fugacity $\lambda_{i}^{g}=0.5$, and for light quark fugacity $\lambda_{i}^{q}=\frac{1}{5} \lambda_{i}^{g}$ of the parton plasma formed at RHIC (LHC) at initial time $\tau_{i}=0.2 \mathrm{fm} / \mathrm{c}$. To achieve an upper "optimistic" limit of the thermal dilepton yield we consider below also higher values of the initial quark and gluon fugacities $\left(\lambda_{i}^{q}=0.5,1\right.$, and $\left.\lambda_{i}^{g}=1\right)$ which lead to different final states. For the sake of definiteness we assume full saturation at confinement temperature $T_{c}=$ $170 \mathrm{MeV}$ and a quadratic time dependence of $\lambda^{q, g}(\tau)[15,24]$.

To get realistic dilepton spectra which can be measured in relativistic heavy-ion collisions one should take into account the experimental conditions related to the acceptance of detectors. To do so we employ Monte Carlo simulations with proper distributions of created leptons with respect to individual rapidities $y_{ \pm}$and transverse momenta $\vec{p}_{\perp \pm}$. In case of the thermal emission of leptons from deconfined matter the needed distribution can be obtained directly from eq. (3). Due to the energymomentum conservation the exponential function, originating from the Boltzmann distribution, can be rewritten as $\exp \left\{\left(p_{1}+p_{2}\right) \cdot u / T\right\}=\exp \left\{\left(p_{+}+p_{-}\right) \cdot u / T\right\}$. Since also $\left|\mathcal{M}\left(12 \rightarrow e^{+} e^{-}\right)\right|^{2}=\left|\mathcal{M}\left(e^{+} e^{-} \rightarrow 12\right)\right|^{2}$ the integration in eq. (3) over the parton momenta yields 


$$
\frac{d N_{e^{+} e^{-}}}{d^{4} x}=\int \frac{d^{3} p_{+}}{2 E_{+}(2 \pi)^{3}} \frac{d^{3} p_{-}}{2 E_{-}(2 \pi)^{3}} \exp \left\{-\frac{\left(p_{+}+p_{-}\right) \cdot u}{T}\right\} F \sigma\left(e^{+} e^{-} \rightarrow 12\right),
$$

where $\sigma\left(e^{+} e^{-} \rightarrow 12\right)=\sigma\left(12 \rightarrow e^{+} e^{-}\right)=\sigma\left(M^{2}\right)$ and the flux factor becomes $F=2 M^{2}$. Then the space-time integrated distribution of thermal leptons reads

$$
\frac{d N_{e^{+} e^{-}}}{d^{2} p_{+} d^{2} p_{-} d y_{+} d y_{-}}=\frac{\alpha^{2} R_{A}^{2}}{4 \pi^{5}} F_{q} \int d \tau \tau K_{0}\left(\frac{M_{\perp}}{T(\tau)}\right) \lambda_{q}^{2}(\tau)
$$

with dilepton transverse mass $M_{\perp}^{2}=p_{\perp_{+}}^{2}+p_{\perp_{-}}^{2}+2 p_{\perp_{+}} p_{\perp_{-}-} \operatorname{ch}\left(y_{+}-y_{-}\right)$. To get the invariant mass spectra we also use the kinematical relationship $M^{2}=2 p_{\perp_{+}} p_{\perp_{-}}\left[\mathrm{ch}\left(y_{+}-\right.\right.$ $\left.\left.y_{-}\right)-\cos \left(\phi_{+}-\phi_{-}\right)\right]$, where $\phi_{ \pm}$denote the azimuthal angles of the leptons in the transverse plane.

As pointed out above, due to the dominant contribution of correlated open charm and bottom decays via $D(B) \bar{D}(\vec{B}) \rightarrow e^{+} X e^{-\bar{X}}$ into the high invariant mass dilepton spectra it is very unlikely that the thermal dilepton production from quark fusion can be observed by using the distribution $d N_{e^{+} e^{-}} / d M^{2}$ alone even when imposing tricky cuts. In this situation the more detailed information of the full spectrum $d N_{e^{+} e^{-}} / d M_{\perp}^{2} d Q_{\perp}^{2} d Y$ is expected to allow for better chances to extract the wanted thermal signal.

As known since some time $[24,29,30]$ and as directly seen in eq. (6) the dilepton yield from an equilibrium quark-gluon plasma depends only on the transverse mass, i.e., $d N_{e^{+} e^{-}} / d M_{\perp}^{2} d Q_{\perp}^{2} d Y \propto f\left(M_{\perp}\right)$; that is, it scales with $M_{\perp}$. The main conditions for this famous $M_{\perp}$ scaling are [29] (i) local thermalization of the source of the dileptons, (ii) no scale other then the temperature, and (iii) predominant boost-invariant flow of matter. The most convincing way to see the validity of the $M_{\perp}$ scaling is to consider the $Q_{\perp}$ dependence of the dilepton spectrum at fixed value of $M_{\perp}$. In case of the boostinvariantly expanding quark-gluon plasma one gets for $d N_{e^{+} e^{-}} /\left.d M_{\perp}^{2}\right|_{M_{\perp}=\text { fix }} d Q_{\perp}^{2} d Y$ indeed a straight line between $Q_{\perp}=0$ and $M_{\perp}$. A detailed analysis of the transverse momentum dependence of the dilepton spectra is performed in ref. [24], where the possible origins of a $M_{\perp}$ scaling violation related to transverse flow and contributions from the hadron gas are studied. The transverse expansion of deconfined matter affects hardly the $M_{\perp}$ scaling, while for hadron matter the life time is strongly reduced, so that its contribution to the total spectrum is moderate. In fact, our previous studies [24] show that the conditions for the $\dot{M}_{\perp}$ scaling are satisfied for radiation from thermalized matter expected to be created in central collisions at RHIC and LHC. At the same time the dileptons from correlated open charm and bottom decay should strongly violate the $M_{\perp}$ scaling if the $c \bar{c}$ and $b \bar{b}$ pairs are dominantly produced by hard initial parton 
collisions. The qualitative argument is as follows: Similar to the Drell-Yan process the hard charm and bottom pairs are created mainly back-to-back (unless hard gluon radiation spoils this correlation), i.e., the pair momentum is preferential $Q_{\perp}^{Q \bar{Q}} \approx 0$, and consequently also the $Q_{\perp}^{Q \bar{Q}}$ spectrum at fixed $M_{\perp}^{Q \bar{Q}}$ is strongly peaked at 0 . The subsequent semileptonic decays cause a partial randomization of the lepton directions but, due to the Lorentz boost effect, both the leptons still are concentrated at small values of $Q_{\perp}$. As a result one can expect that in the large $Q_{\perp}$ region the thermal signal sticks out, while the semileptonic decay products are sharply dropping.

\section{Monte Carlo simulations}

To quantify this effect we perform Monte Carlo calculations generating thermal dielectrons in accordance with the distribution eq. (9). As above we take into account the acceptances of PHENIX at RHIC and ALICE at LHC. First we consider the simplified case without any momentum degradation of heavy quarks, i.e., energy loss effects are not included and the $\delta$ function fragmentation scheme is employed. This is obviously the most favorable case for the relative contribution from decay dileptons to the full dilepton yield.

In order to get some threshold effect for the decay dileptons in dependence of $Q_{\perp}$ at fixed $M_{\perp}$ one needs to select leptons with individual transverse momenta greater than the maximum electron energy in the rest system of the decaying open charm or bottom meson. Typically such a threshold value is given by 1 (2) GeV for charm (bottom). The results of our calculations of the $Q_{\perp}$ spectra for RHIC conditions are displayed in fig. 3a. We select here dileptons with transverse mass within the interval $2 \mathrm{GeV}<M_{\perp}<3 \mathrm{GeV}$. The value of the minimum transverse momentum of leptons is chosen as $p_{\perp}^{\mathrm{min}}=1 \mathrm{GeV}$. The detector acceptance destroys the ideal $M_{\perp}$ scaling, i.e., the thermal yield gets some structure reflecting the $p_{\perp}$ cut and the geometry. One can see the threshold like behavior of the dilepton spectrum from charm decays: there is a rapid dropping of the yield at $Q_{\perp}>1.3 \mathrm{GeV}$. On the contrary the thermal dilepton spectrum sticks out up to the kinematical boundary $Q_{\perp}=M_{\perp}$. Unfortunately such a value of $p_{\perp}^{\min }=1 \mathrm{GeV}$ is not large enough to cause a similar suppression effect for leptons from bottom decays. Nevertheless as pointed out in ref. [7] and as seen in fig. $3 \mathrm{a}$ the contribution from bottom decays is suppressed (this is due to the narrow rapidity gap covered by PHENIX) but it is clearly above the thermal yield for the initial quark fugacity $\lambda_{q}=0.1$. To estimate the chances to see a thermal dilepton signal over the 
background from bottom decays we perform variations of the initial quark fugacity. Due to the factor $\lambda_{q}^{2}$ in the thermal rate eq. (9), even a comparatively small increase of the initial quark phase space saturation can result in a noticeable enhancement of thermal dileptons compared to the decay background. This can be seen in fig. 3a for the initial quark fugacities $\lambda_{q}=0.5$ and 1 . For the latter value the thermal signal is above the bottom decay contribution.

For LHC conditions one can obtain the threshold like effect in the $Q_{\perp}$ spectrum even for the dielectrons from the massive bottom decays. In fig. $3 b$ we show our results for dileptons within the transverse mass interval $5.25<M_{\perp}<5.75 \mathrm{GeV}$. The electron transverse momentum cut $p_{\perp}^{\mathrm{min}}=2 \mathrm{GeV}$ causes a sufficiently strong threshold effect both for charm and bottom, while the dielectrons from thermalized matter exhibit the approximate $M_{\perp}$ scaling even for such a value of $p_{\perp}^{\min }$. It gives therefore the opportunity to measure the thermal dilepton signal on the basis of the $M_{\perp}$ scaling at LHC conditions. Actually such measurements could be performed for lepton pairs with high enough transverse momentum but smaller invariant mass. In particular, as follows from fig. $3 \mathrm{~b}$, for a fixed value of $M_{\perp} \simeq 5.5 \mathrm{GeV}$ the proper region for transverse pair momenta is about $4 \mathrm{GeV}<Q_{\perp}<5.5 \mathrm{GeV}$. This window increases considerably with increasing initial quark fugacity $\lambda_{q}^{i}$ of thermalized parton matter.

We perform also calculations of the $Q_{\perp}$ spectra at fixed $M_{\perp}$ for decay dileptons including the Peterson fragmentation function [31], where the function

$$
f(z)=\left[z\left(1-\frac{1}{z}-\frac{\epsilon}{1-z}\right)^{2}\right]^{-1}
$$

( $\epsilon=0.06(0.02)$ for charm (bottom) [32]) prescribes the probability to change the spatial momentum of a heavy quark from $\vec{p}$ to $z \vec{p}$ during hadronization. Independently we also study the influence of energy loss of heavy quarks in the deconfined medium. Both these effects cause a suppression of the dilepton yield from open charm and bottom, in particular in the small- $Q_{\perp}$ (large- $M$ ) region. Since the suppression effect due to Peterson's fragmentation is quite small (typically within factor 2) we do not display it separately. On the contrary, the degradation of the heavy quark momenta due to energy loss in the deconfined medium causes a considerably suppression of the contribution of decay leptons in the $Q_{\perp}$ spectrum at fixed value of $M_{\perp}$. In fig. 4 we show the results of our calculations for $\mathrm{LHC}$ conditions with the same kinematical cuts as in fig. $3 \mathrm{~b}$. As seen in this plot, both charm and bottom are considerably suppressed due to energy loss effects, in particular in the region of smaller values of $Q_{\perp}$. These calculations exploit the model I. In case of model II the suppression effect is much 
stronger. With respect to the wanted thermal dilepton signal the suppression of the $Q_{\perp}$ spectra from bottom decays appears most important. The proper $Q_{\perp}$ window to extract thermal dileptons becomes greater, e.g., $3 \mathrm{GeV}<Q_{\perp}<5.5 \mathrm{GeV}$ for initial quark fugacity $\lambda_{q}=0.5$. Therefore, the energy loss effect obviously helps to measure thermal dielectrons with ALICE at LHC on the basis of the $M_{\perp}$ scaling behavior.

To clarify whether the proposed signal is experimentally feasible we estimate the background from $\pi^{0}$ decays within the same kinematical gates. For this background study we also perform Monte Carlo simulations of $\pi^{0} \rightarrow e^{+} e^{-} \gamma$ decays utilizing the distribution $d N_{\pi^{0}} / d y d p_{\perp}=\left(d N_{\pi^{0}} / d y\right) \exp \left\{p_{\perp} / T_{\pi}\right\}$ with $T_{\pi}=200 \mathrm{MeV}$ in accordance with present SPS results for central $\mathrm{Pb}+\mathrm{Pb}$ collisions at $158 \mathrm{AGeV}$ beam energies [33]. The slope parameter $T_{\pi}$ is a convolution of local freeze-out temperature and transverse flow. The latter one is expected to increase from SPS to RHIC to LHC due to higher rapidity densities, and it might cause a slightly higher value of $T_{\pi}$. We choose here the fictitious values $d N_{\pi^{0}} / d y=2500$ (500) for LHC (RHIC). Each $\pi^{0}$ is three-body decayed in its rest frame with the branching ratio of $1.2 \%$ [22] and then the resulting $e^{+} e^{-}$pair is boosted and filtered. We find the individual $\pi^{0}$ decay dileptons with 5.25 $\mathrm{GeV}<M_{\perp}<5.75 \mathrm{GeV}$ three orders of magnitude below the thermal dilepton signal and only at $Q_{\perp}>5.2 \mathrm{GeV}$. The flattening of the pion spectrum at large values of $p_{\perp}$, due the Cronin effect and hard scatterings, is not covered by our parametrization of the $p_{\perp}^{\pi^{0}}$ spectrum; it will enhance the pion decay contribution. Otherwise some quenching [12] at large values of $p_{\perp}^{\pi^{0}}$ can counteract and balance such effects. The finite experimental momentum resolution will cause a decay contribution also at somewhat smaller values of $Q_{\perp}$. However, these effects will hardly close the window for the thermal signal. The next-to-leading order Drell-Yan contribution is also estimated to be strongly suppressed at larger values of $Q_{\perp}$ (say, for $Q_{\perp}>1 \mathrm{GeV}$ ) because of the chosen large $p_{\perp}$ cut. The most prominent danger however comes from the combinatorical background from uncorrelated hadron decays: Only after experimentally removing this background together with the background from the conversion dileptons, emerging from energetic photons in the detector material, one has a chance to see the interesting signal.

Fully analog considerations also apply at RHIC. However, selecting electron pairs with such large values of $M_{\perp} \approx 5.5 \mathrm{GeV}$ and choosing $p_{\perp}^{\mathrm{min}}=2 \mathrm{GeV}$ results in prohibitory small counting rates which seem to make such a strategy not feasible. For the above choice of $M_{\perp} \approx 2.5 \mathrm{GeV}$ and choosing $p_{\perp}^{\min }=1 \mathrm{GeV}$ the dileptons from $\pi^{0}$ decays produce here a contribution which is three orders of magnitude above the 
thermal signal, however only in the interval $Q_{\perp}>1.95 \mathrm{GeV}$. Therefore, one remains in a situation as depicted in fig. $3 \mathrm{a}$ and discussed above.

\section{CONCLUSIONS}

In summary we analyze the chances of observing a thermal dilepton signal in highenergy heavy-ion collisions. Exploiting currently estimated energy loss effects of heavy quarks passing through deconfined matter we find that the invariant mass spectra of dileptons, as measurable in the detector facilities P.HENIX and ALICE, offer hardly a chance to find thermal dileptons with $M>2 \mathrm{GeV}$. Even if in optimistic energy loss scenarios the dileptons from correlated charm and bottom decays compete with the Drell-Yan background, the thermal signal is significantly smaller. Since the energy loss is a poorly quantified effect we stress the need to make attempts to verify it, for instance via the single electron $p_{\perp}$ spectra which should reflect the transverse momentum degradation of parent charm and bottom mesons.

The double differential rate $d N_{e^{+} e^{-}} / d M_{\perp}^{2} d Q_{\perp}^{2}$ with $M_{\perp}$ in a narrow interval and with a suitable $p_{\perp}^{\min }$ cut on the individual leptons, however, seems to allow for a window at large values of the pair transverse momentum where the thermal yield shines out. At RHIC this is probably not feasible, while at LHC the estimated high initial temperature of the parton matter causes a stronger thermal signal. The basic issue is here whether the combinatorical background of many other strong sources can be subtracted with sufficient accuracy.

\section{Acknowledgments}

Stimulating discussions with R. Baier, E.V. Shuryak, M. Tannenbaum, R. Vogt, X.N. Wang, and G.M. Zinovjev are gratefully acknowledged. O.P.P. thanks for the warm hospitality of the nuclear theory group in the Research Center Rossendorf. This work is supported in parts by BMBF grant 06DR829/1 and USFFR grant 2.5.1/41. 
[1] E.V. Shuryak, Phys. Lett. B 78 (1978) 150

[2] PHENIX Conceptúal Design Report, 1993, unpublished

[3] ALICE Technical Proposal, CERN/LHCC/ 95-71, 1995

[4] G. Agakishev et al. (CERES collaboration), Phys. Rev. Lett. 75 (1995) 1272

[5] M. Gonin et al. (NA50 collaboration), Nucl. Phys. A 610 (1996) 404c

[6] A. Drees, Nucl. Phys. A 610 (1996) 536c

[7] S. Gavin, P.L. McGaughey, P.V. Ruuskanen, and R. Vogt, Phys. Rev. C 54 (1996) 2606

[8] E.V. Shuryak, Phys. Rev. C 55 (1997) 961

[9] B. Kämpfer, O.P. Pavlenko, and K. Gallmeister, FZR-188 (1997), Phys. Lett. in print

[10] Z. Lin, R. Vogt, and X.N. Wang, nucl-th/9705006, Phys. Rev. C in print

[11] R. Baier, private communication, R. Baier, Yu.L. Dokshitzer, A.H. Mueller, and D. Schiff, to be published

[12] X.N. Wang, and Z. Huang, Phys. Rev. C 55 (1997) 3047

[13] B. Svetitsky, and A. Uziel, Phys. Rev. D 55 (1997) 2616

[14] B. Kämpfer, O.P. Pavlenko, A. Peshier, M. Hentschel, and G. Soff, J. Phys. G 23 (1997) 2001

[15] B. Kämpfer, and O.P. Pavlenko. Phys. Lett. B 391 (1997) 185

[16] A.D. Martins, W.J. Stirling, and R.G. Roberts, Phys. Lett. B 306 (1993) 145

[17] V. Emel'yanov, A. Khodinov, S.R. Klein, R. Vogt, nucl-th/9706085

[18] M.G. Mustafa, D. Pal, and D.K. Srivastava, nucl-th/9706001, Phys. Rev. C in print, M.G. Mustafa, D. Pal, D.K. Srivastava, and M. Thoma, nucl-th/9711059

[19] R. Baier, Yu.L. Dokshitzer, S. Peigne, and D. Schiff, Phys. Lett. B 345 (1995) 277

[20] R. Baier, Yu.L. Dokshitzer, A.H. Mueller, S. Peigne, and D. Schiff, Nucl. Phys. B 483 (1997) 291, В 484 (1997) 265

[21] B.G. Zakharov, hep-th/9704255 
[22] R.M. Barnett et al. (Particle Data Group), Phys. Rev. D 54 (1996) 1

[23] M. Tannenbaum, Heavy Ion Physics 4 (1996) 139

[24] B. Kämpfer, O.P. Pavlenko, A. Peshier, and G. Soff, Phys. Rev. C 52 (1995) 2704

[25] P.V. Ruuskanen, in Quark Gluon Plasma, World Scientific, Singapore (1990), (ed.) R. Hwa, p. 519

[26] T.S. Biro,-E. van Doorn, B. Müller, M.H. Thoma, and X.-N. Wang, Phys. Rev. C 48 (1993) 1275 ,

P. Levai, B. Müller and X.-N. Wang, Phys. Rev. C 51 (1995) 3326

[27] K.J. Eskola, K. Kajantie, and P.V. Ruuskanen, Phys. Lett. B 332 (1994) 191, K.J. Eskola, Nucl. Phys. A 590 (1995) 383c

[28] K.J. Eskola, B. Müller, and X.-N. Wang, Phys. Lett. B 374 (1996) 20

[29] L. McLerran, and T. Toimela, Phys. Rev. D 31 (1985) 545

[30] M. Asakawa, C.M. Ko, and P. Levai, Phys. Rev. Lett. 70 (1993) 398

[31] C. Peterson, D. Schlatter, I. Schmitt, and P.M. Zerwas, Phys. Rev. D 27 (1983) 105

[32] M. Cacciari, hep-ph/9708282

[33] P.G. Jones et al. (NA49 collaboration), Nucl. Phys. A 610 (1996) 188c 
RHIC

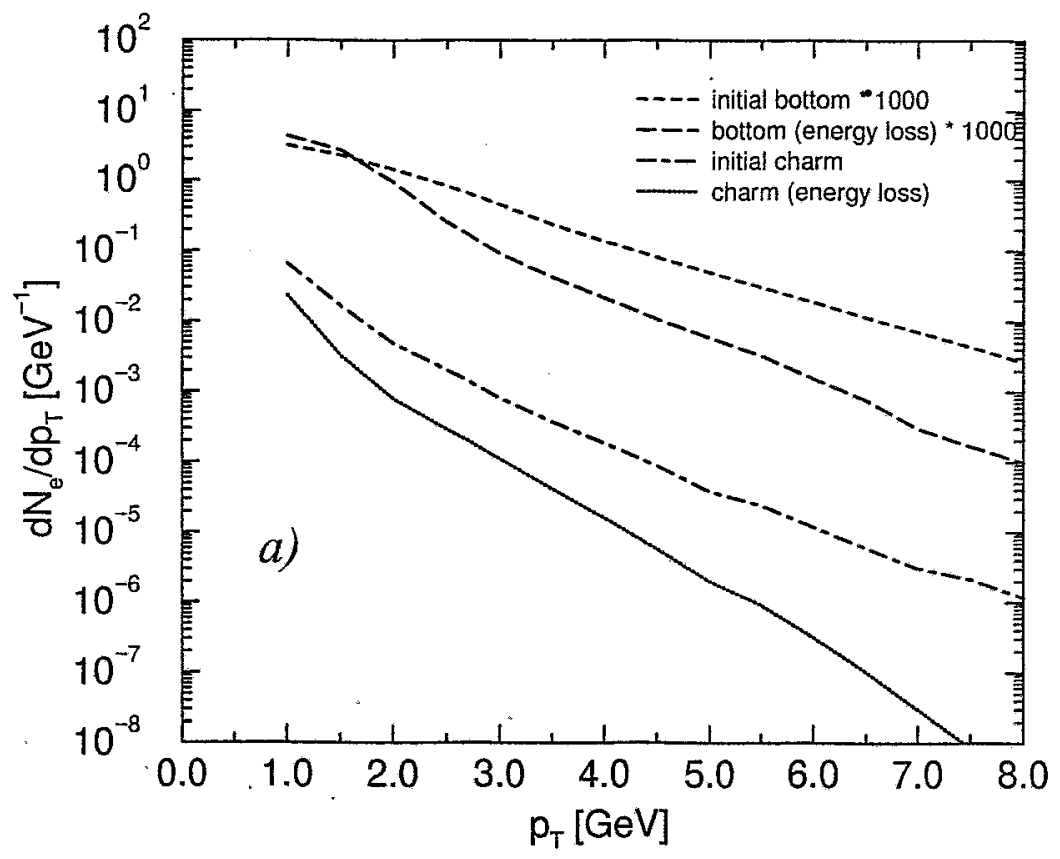

RHIC

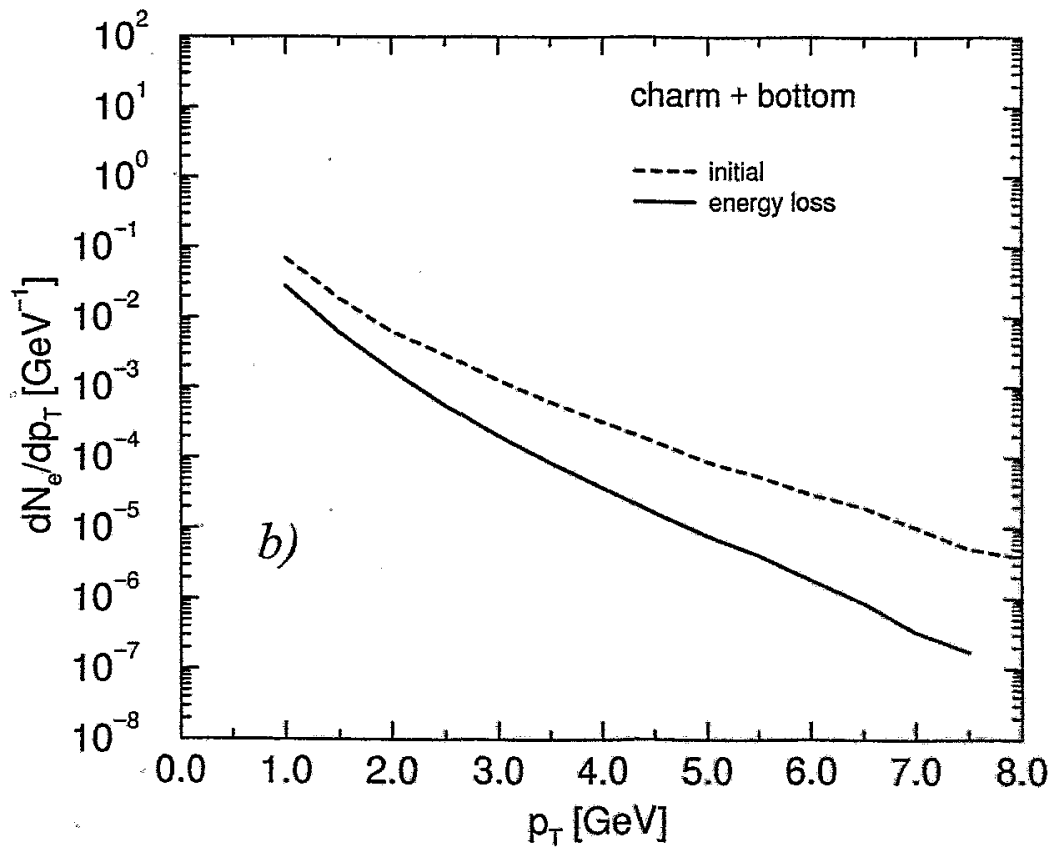

FIG. 1. The transverse momentum spectra of single electrons from $D$ and $B$ meson decays (a) and their sum (b) at RHIC energies within the PHENIX acceptance. Displayed are the spectra before ("initial") and after energy loss within model II. 
RHIC

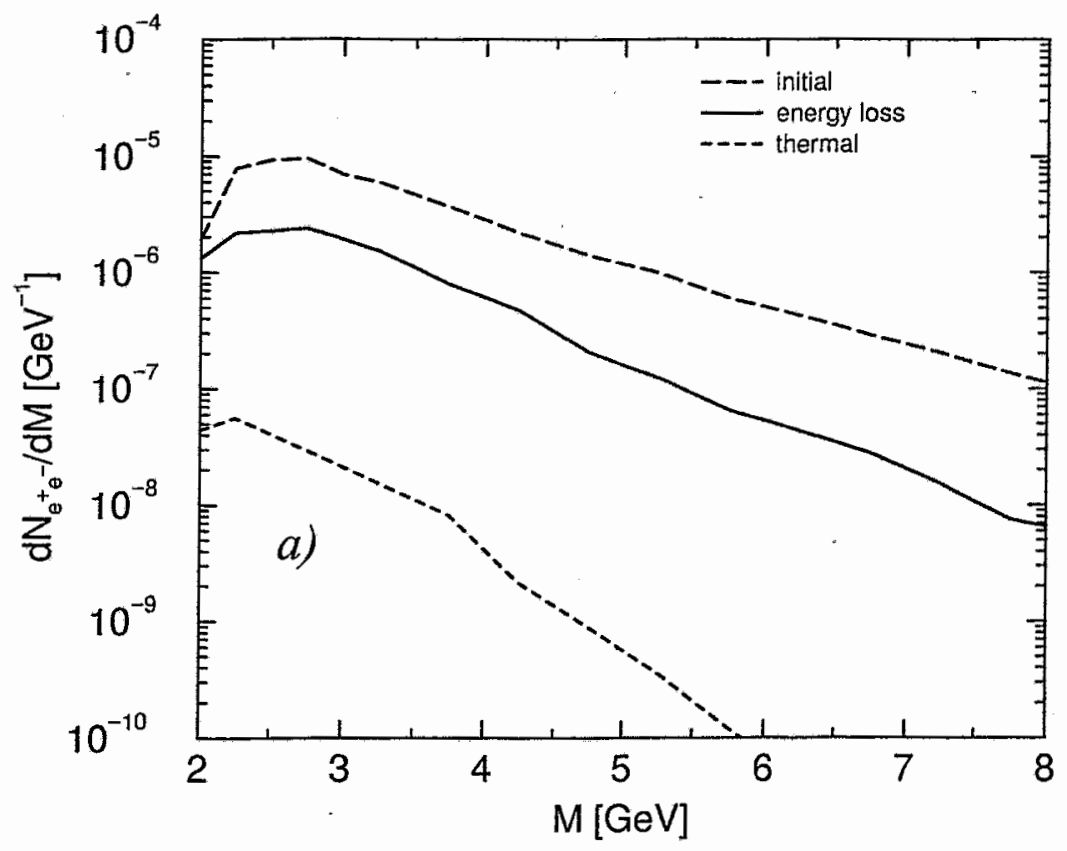

LHC

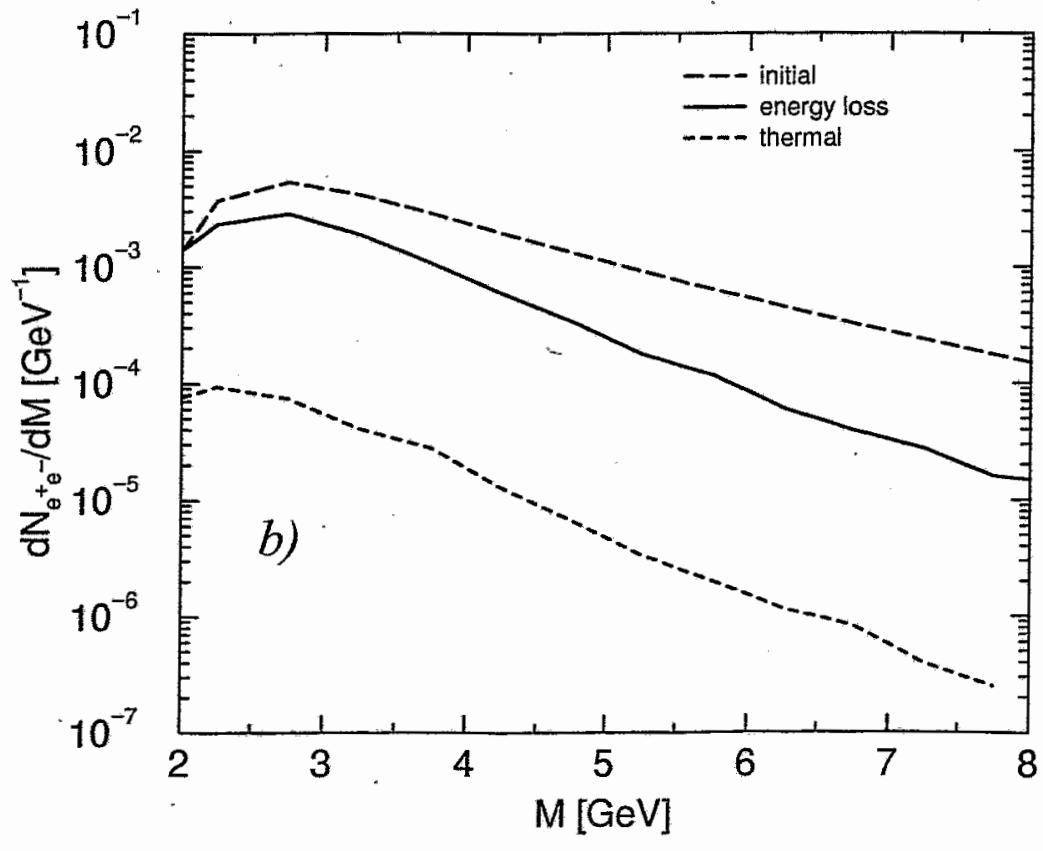

FIG. 2. Dielectron spectra from correlated charm and bottom decays as a function of the invariant mass; long-dashed (solid) lines are for results without (with) energy loss according to model II for RHIC with PHENIX acceptance (a) and model I for LHC with ALICE acceptance but without high- $p_{\perp}$ cut (b). The dashed lines depict the thermal dielectron spectrum. For the strength of the Drell-Yan contribution cf. [9,10] 
RHIC

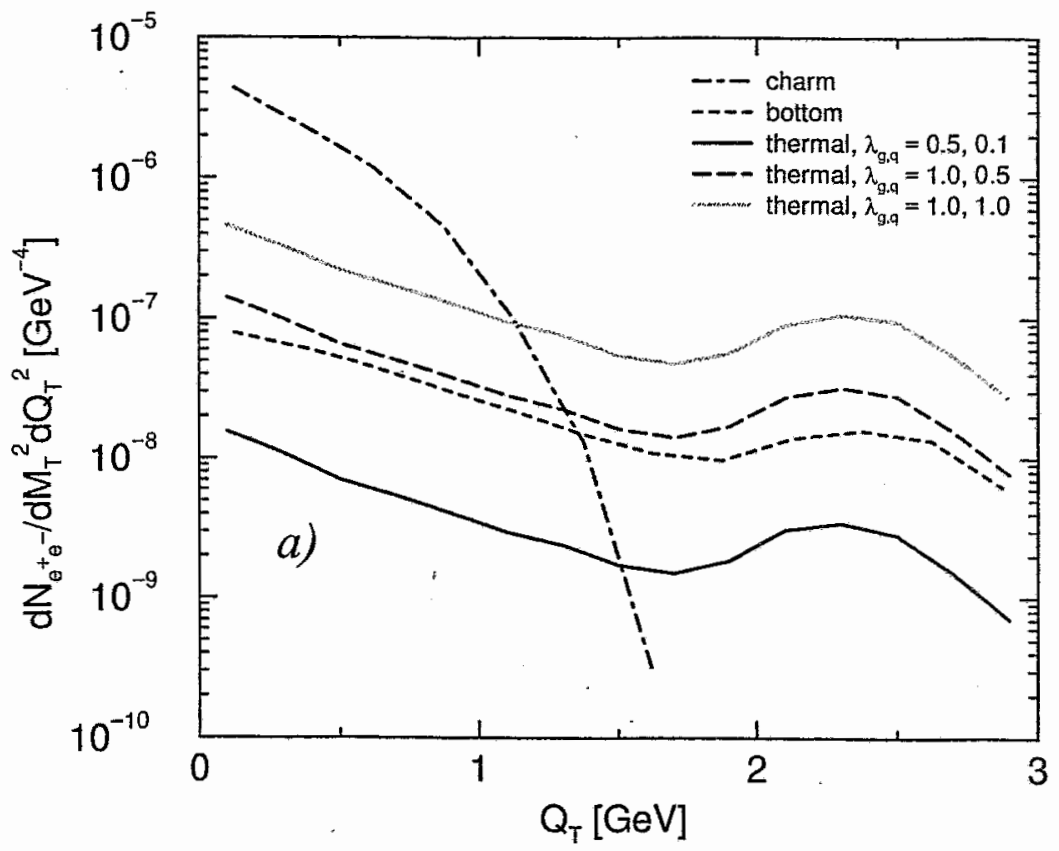

LHC

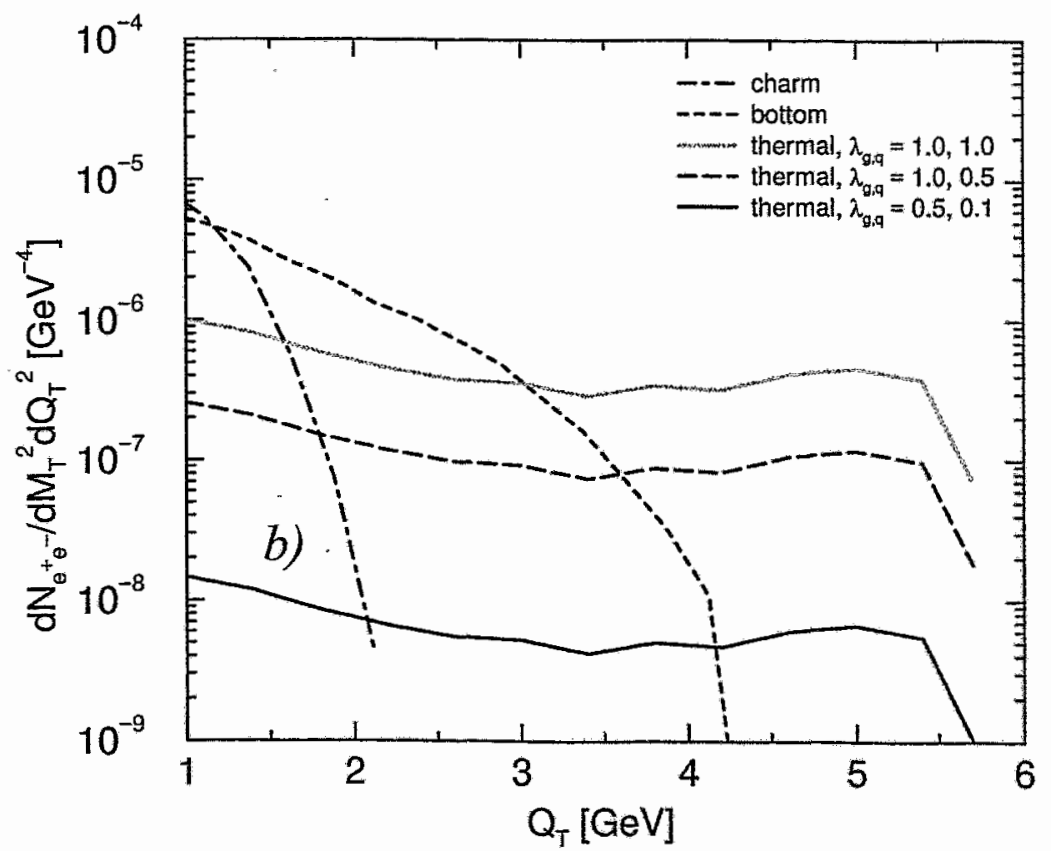

FIG. 3. The transverse momentum spectra of dielectrons at constrained transverse masses:

(a) RHIC with PHENIX acceptance, $p_{\perp}>1 \mathrm{GeV}$ and $2 \mathrm{GeV}<M_{\perp}<3 \mathrm{GeV}$, (b) LHC with ALICE acceptance, $p_{\perp}>2 \mathrm{GeV}$ and $5.25 \mathrm{GeV}<M_{\perp}<5.75 \mathrm{GeV}$. The dash-dotted (short-dashed) lines are for correlated charm (bottom) decays. The full, long-dashed and gray-dotted lines depict the thermal yield for various initial quark and gluon fugacities. 


\section{LHC}

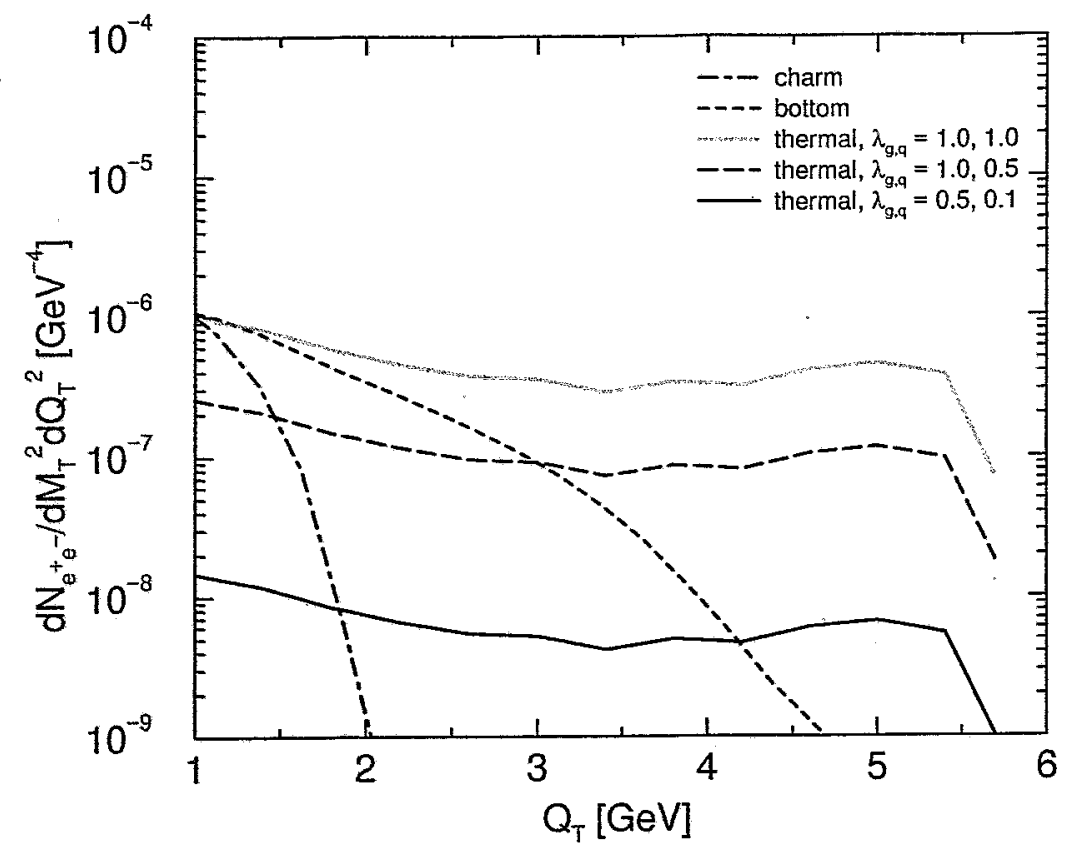

FIG. 4. The same as in fig. $3 \mathrm{~b}$ but with energy loss effects according to model I. 